\title{
Student difficulties with quantum uncertainty in the context of discrete probability distributions
}

\author{
Yujia Li and Antje Kohnle \\ School of Physics and Astronomy, University of St Andrews, St Andrews, KY16 9SS, United Kingdom \\ Gina Passante \\ Department of Physics, California State University Fullerton, Fullerton, CA 92831, USA
}

Quantum uncertainty is a fundamental concept in quantum mechanics, but challenging for students to master. In this article, we describe student difficulties with visual and conceptual understanding of quantum uncertainty in the context of discrete probability distributions such as those for a spin $1 / 2$ particle. We collected written responses from students at two institutions to a homework activity focusing on uncertainty of spin measurement outcomes, as well as written responses to a test question from one of the institutions. We also conducted interviews with six students to gain further insight into difficulties found. Common incorrect ideas found included a depiction of uncertainty as the error around each of the individual measurement outcomes, not depicting the uncertainty region from the expectation value outwards, and the idea that quantum uncertainty of an observable can never be zero. These ideas may indicate a confusion between quantum uncertainty and errors due to instrumental imperfections of the measurement apparatus, a lack of conceptual understanding of quantum uncertainty as the standard deviation of the probability distribution with respect to its mean, and an incorrect interpretation of the uncertainty relation between two incompatible observables to deduce that quantum uncertainty can never be zero. The results of this study show the importance of supporting students in visual and conceptual understanding of quantum uncertainty.

2021 PERC Proceedings edited by Bennett, Frank, and Vieyra; Peer-reviewed, doi.org/10.1119/perc.2021.pr.Li Published by the American Association of Physics Teachers under a Creative Commons Attribution 4.0 license. Further distribution must maintain the cover page and attribution to the article's authors. 


\section{INTRODUCTION}

The difference between classical ignorance and quantum uncertainty is a fundamental concept in quantum mechanics, but challenging for students to master. In quantum mechanics, states are characterized by probability distributions with respect to measurement outcomes of a given observable, and these probability distributions are intrinsic to the quantum state. In contrast, classical ignorance is due to our lack of knowledge of a definite state.

For a given quantum state $|\psi\rangle$ and an observable $\hat{Q}$ with discrete eigenvalues $q_{n}$, the quantum uncertainty $\Delta Q$ is the standard deviation of the probability distribution prior to measurement of the outcomes $q_{n}$ with respect to $|\psi\rangle$. Mathematically, this uncertainty is the sum of the measurement outcomes weighted by their respective probabilities, and can be obtained via the expectation values of the operator and its square with respect to the given $|\psi\rangle$. The uncertainty $\Delta Q>0$ for superposition states and $\Delta Q=0$ for an eigenstate with respect to $\hat{Q}$. If we make a measurement of the observable represented by $\hat{Q}$ on an initial superposition state, then this collapses the system to one of the eigenstates in a non-deterministic way.

In this study, we are interested in students' conceptual and visual understanding of quantum uncertainty in the context of discrete probability distributions, such as those for the spin components of a spin 1/2 particle. Given its fundamental importance for quantum mechanics, a correct conceptual and visual understanding of quantum uncertainty seems a key instructional goal.

Studies in quantum mechanics research have found that students can be proficient in calculations but lack conceptual and visual understanding of the underlying concepts (e.g. [1]). Prior work focusing on student understanding of quantum expectation values found that students rarely reason conceptually via the expectation value as the average of a large number of single measurements on identically prepared systems [2]. Previous investigations have studied students' understanding of the Heisenberg Uncertainty Principle, e.g. uncertainty in the context of continuous distributions of position and momentum. Common incorrect ideas were that uncertainty is due to external effects, measurement errors or measurement disturbance [3-6]. Recent work comparing student interpretation of uncertainty in classical and quantum mechanics experiments found that students often did not consider the influence of experimental error [7, 8] but rather only the inherent quantum uncertainty due to the probabilistic nature of the theory. However, this result depended on context, with students also considering experimental errors for a spectrum experiment [8].

This article is structured as follows: Section II describes the data collection of written responses, their analysis and the interviews. Section III describes three common difficulties found in the written responses and discusses them in light of the interviews. Finally, section IV summarizes the difficulties found and their implications.

\section{DATA COLLECTION AND METHODS}

In this study, we collected written responses from students at two institutions, the University of St Andrews (StA) in the UK and California State University Fullerton (CSUF) in USA. Responses were collected in junior-level quantum mechanics courses which covered spin and Stern-Gerlach experiments. At CSUF, the course follows a spins-first approach, with the postulates of quantum mechanics derived from spin 1/2 particles and Stern-Gerlach experiments. StA follows a wave mechanics approach, with spin only discussed towards the end of the second semester of the two-semester sequence.

In order to probe students' visual understanding of quantum uncertainty, we collected written responses from students at CSUF $(N=24)$ and StA $(N=86)$ to a combined simulationtutorial activity on the topic of quantum uncertainty in the context of spin 1/2. In the so-called simulation-tutorial activities, students first work on questions without simulation support, and in a second phase work on further questions after playing with a simulation $[9,10]$. This activity used a simulation "Uncertainty of spin measurement outcomes" [11]. The activity was given as a homework assignment at both institutions.

In the first part of the activity without the simulation, students are asked to consider a setup with two successive SternGerlach apparatuses (SGAs). The first SGA is oriented along $Z$ to prepare the spin state $\left|\uparrow_{z}\right\rangle$ (spin-up along $Z$ ), and the second SGA is oriented along $X$, e.g. at an angle $\theta=90^{\circ}$ with respect to the $Z$ direction. Students are asked to determine the expectation value $\left\langle\hat{S}_{x}\right\rangle$ and the quantum uncertainty $\Delta S_{x}$ for the second SGA and to make an outcome histogram including these quantities.

We are here interested in one of the activity tasks in the second phase with the simulation, shown in Fig. 1a. In this task, students are asked to consider the same setup with two Stern-Gerlach apparatuses (SGAs), but the second SGA is now oriented at an angle $\theta=60^{\circ}$ with respect to the $Z$ direction. The simulation allows students to rotate the second SGA. Students are asked to make a histogram of the outcome probabilities and the quantum uncertainty, with these values and a histogram of the outcomes being provided by the simulation. Importantly, the expectation value and uncertainty are not shown graphically in the simulation, to allow us to probe students' visual understanding of these quantities. Students could read off the simulation that for $\theta=60^{\circ}$, the probabilities for the outcomes $+\hbar / 2$ and $-\hbar / 2$ are 0.75 and 0.25 respectively, the expectation value $\left\langle\hat{S}_{\theta}\right\rangle=0.25 \hbar$ and the quantum uncertainty $\Delta S_{\theta}=0.433 \hbar$. Fig. 1 shows the histogram with these values included.

Not all students included the uncertainty in their histogram; in total there were $13 \mathrm{CSUF}$ and $73 \mathrm{StA}$ responses with graphical representations of the uncertainty for this task. This corresponded to $54 \%$ of CSUF responses and $85 \%$ of StA responses. We coded students' sketches with respect to the content, focussing on domain accuracy or inaccuracy to identify incorrect ideas. We were particularly interested in how 
a) Consider the value of angle $\theta=60^{\circ}$ for the second Stern-Gerlach apparatus. Reading off the values given in the simulation, sketch a histogram of the probabilities for the different outcomes. Indicate $\Delta S_{60}$ on your histogram. Carefully label your histogram!

Solution to a)

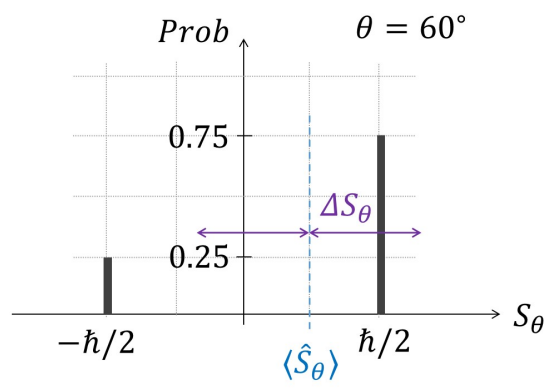

b) Consider a general operator $\hat{Q}$ for a one-dimensional system. Assume that $\psi_{n}$ is a normalized eigenfunction of $\hat{Q}$ with eigenvalue $q_{n}$. Calculate the uncertainty $\Delta Q$ with respect to $\psi_{n}$. Briefly interpret your result.

FIG. 1. (a) One of the tasks in the simulation-tutorial activity focusing on visual understanding of quantum uncertainty (sentence in italics), and its solution. (b) A mid-term test question. This study focuses on the final conceptual part (in italics), where students are asked to interpret their result for the uncertainty. Sentences in italics are for emphasis here, and were not italicized in the original versions.

the quantum uncertainty was depicted in students' histograms and so focussed on this feature when creating the codes. We also coded whether or not the expectation value was included in the sketch, and whether the uncertainty region extended only in one direction or in two directions from some middle value.

In order to probe students' conceptual understanding of quantum uncertainty, we also collected written responses from a mid-term test question shown in Fig. 1b. This question was only administered to StA students $(N=107)$ in another academic session, so a different year group to that of the activity question shown in Fig. 1a. For this test question (Fig. 1b), students are asked to determine the uncertainty $\Delta Q$ with respect to an eigenfunction $\psi_{n}$ of a general operator $\hat{Q}$ with discrete eigenvalues. For this case, the only possible outcome is the eigenvalue $q_{n}$, so that the outcome is $100 \%$ certain prior to measurement. Thus, $\Delta Q=0$. This is also seen mathematically, as $\langle\hat{Q}\rangle=\left\langle\psi_{n}|\hat{Q}| \psi_{n}\right\rangle=q_{n}\left\langle\psi_{n} \mid \psi_{n}\right\rangle=q_{n}$ and $\left\langle\hat{Q}^{2}\right\rangle=\left\langle\psi_{n}\left|\hat{Q}^{2}\right| \psi_{n}\right\rangle=q_{n}^{2}\left\langle\psi_{n} \mid \psi_{n}\right\rangle=q_{n}^{2}$, so that $\Delta Q=\sqrt{q_{n}^{2}-q_{n}^{2}}=0$. In this study, we were interested in students' conceptual understanding of quantum uncertainty rather than their ability to calculate uncertainties. Thus, we

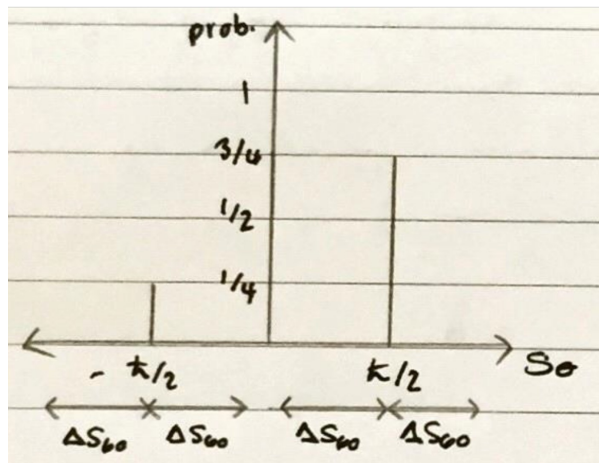

FIG. 2. Sample student response to the activity question (Fig. 1a) showing the incorrect depiction of the quantum uncertainty as the error around each of the measurement outcomes.

only consider here the final part of this question where students are asked to briefly interpret the result that $\Delta Q=0$.

In order to gain further insight into the types of errors found in the written responses, we also conducted individual interviews with six volunteer StA students. These interviews (of one hour duration each) were semi-structured and recorded for further analysis. The interview tasks included making a histogram similar to that shown in Fig. 1a from given values, and asking students to agree or disagree with given statements that related to errors found in the written responses.

\section{OUTCOMES}

In this section we discuss three difficulties found in the analysis of the written responses. We interpret these difficulties in light of the student interviews.

\section{A. Thinking quantum uncertainty is the error around each of the measurement outcomes}

A reasonably common incorrect depiction seen in response to the activity question (Fig. 1a) is shown in Fig. 2. Students sketched the uncertainty region around each of the measurement outcomes individually rather than with respect to the expectation value. This depiction was common at StA, with 19 of 73 students $(26 \%)$ making this error, but not seen in the CSUF depictions. This depiction was associated with not sketching the expectation value, with only 6 of these 19 depictions (32\% of sketches with this error) including $\left\langle\hat{S}_{\theta}\right\rangle$ in their sketch. Students presumably did not add the expectation value due to the uncertainty in their depiction being related to the outcomes and not to the expectation value. This depiction was also associated with sketching the uncertainty region in both directions (in positive and negative directions with respect to each outcome, as seen in Fig. 2), with 16 of $19(84 \%)$ sketches with this error showing $\pm \Delta S_{\theta}$. 


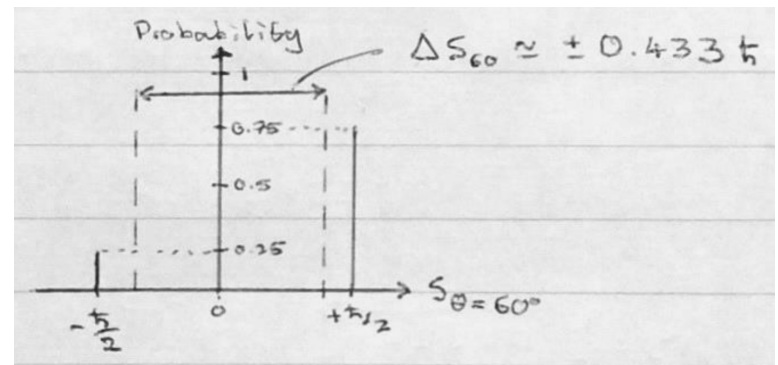

FIG. 3. Sample student response to the activity question (Fig. 1a) showing the quantum uncertainty not being related to the expectation value.

We hypothesized that the reason for this difficulty is a confusion between classical measurement errors due to instrumental imperfections, that would lead to a spread in each of the outcomes obtained in an actual experiment, and the quantum uncertainty that is related to the underlying probability distribution prior to measurement. One would obtain an error around each of the outcomes if one were to perform a Stern-Gerlach experiment and measure the spread of the individual spots for the deflections. This spread would be due to imperfections in the source collimation, the magnetic field configuration, the detector, etc. In contrast to the quantum uncertainty that is a measure of the probability distribution prior to measurement, the errors depicted by these students would be determined after making a set of measurements and due to instrumental imperfections.

One of the six students interviewed also sketched the uncertainty region around each of the outcomes. This student disagreed with the given statement in the interview "The uncertainty value tells us the uncertainty in each of the two measurement outcomes before a measurement", saying "would we only find out the uncertainty after the measurement? I guess.". In response to the interviewer asking "So you think that you can not know the uncertainty before a measurement, but you can know it after a measurement", the student said "Yeah, by finding like the spread". This student then agreed with the given statement "The uncertainty is the error when we measure the outcome". Thus, this student seemed to consider quantum uncertainty as the classical error that can be found after making a set of measurements of the same quantity.

\section{B. Not relating quantum uncertainty to the expectation value}

Quantum uncertainty as the standard deviation of a probability distribution is a measure of the spread of this probability distribution around the mean, e.g. around the expectation value. Hence, the uncertainty region $\Delta S_{\theta}$ in Fig. 1a is depicted with respect to $\left\langle\hat{S}_{\theta}\right\rangle$. A significant number of students' responses to the activity question (Fig. 1a) instead depicted the uncertainty with respect to the origin, either as a range of

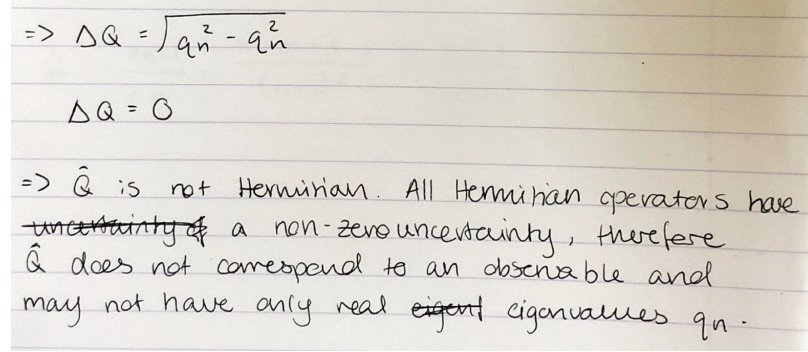

FIG. 4. Sample student response to the final part of the test question shown in Fig. 1b.

values from $S_{\theta}=0$ outwards or as a point on the $S_{\theta}$ axis at the value of $\Delta S_{\theta}$.

23 of 73 StA students (32\%) and 3 of 13 CSUF students (23\%) sketched the uncertainty as a region from zero outwards. An example of this type of depiction is shown in Fig. 3. In addition, 1 of 73 (1\%) StA students and 3 of 13 CSUF students (23\%) sketched the uncertainty as a point on the $S_{\theta}$ axis at the value of $\Delta S_{\theta}$. Only 9 of these 30 responses in total $(30 \%)$ depicted the expectation value $\left\langle\hat{S}_{\theta}\right\rangle$, again indicating that these students did not relate quantum uncertainty to the expectation value. None of the students in the interviews made these types of depictions.

Considering all of the depictions to the activity task shown in Fig. 1a, only 29 of $73 \mathrm{StA}$ students $(40 \%)$ and 4 of 13 CSUF students $(31 \%)$ depicted the uncertainty $\Delta S_{\theta}$ as extending from the expectation value. Thus, the majority of depictions did not consider quantum uncertainty as a measure of the spread around the mean.

\section{Thinking quantum uncertainty in a single observable can not be zero}

We observed this difficulty in the mid-term test question administered at StA (Fig. 1b), as well as in four of the six student interviews. For the test question, 10 of 107 responses (9\%) had an incorrect interpretation in terms of $\hat{Q}$ not being Hermitian (or not corresponding to an observable), as a quantum observable can not have an uncertainty of zero. All of these 10 answers had correctly found that $\Delta Q=0$ in their calculation.

Fig. 4 shows an example student response. Six of these 10 incorrect responses did not justify their reasoning further, similarly to Fig. 4. Two responses explained that an uncertainty of zero was not possible due to the uncertainty principle, e.g. " $\hat{Q}$ does not correspond to a real observable, e.g. as to have 0 uncertainty in one real observable leads to having $\infty$ uncertainty in the corresponding incompatible observable.". These two students seemed to think that infinite uncertainty was not possible. Two further responses justified their reasoning by stating that "exact" or "precise" measurement was not possible. 
We hypothesized that one reason for this difficulty is a confusion between the uncertainty in a single observable and the product of the uncertainties for incompatible observables, as given by an uncertainty relation. Therefore, in the interviews we asked students explicitly whether a quantum uncertainty of zero would violate the uncertainty principle. In response to this question, one of the students said: "OK, so Heisenberg's Uncertainty Principle is saying that you can't be simultaneously certain of a particle's position and momentum, that is, you can't know them both, and there's going to be some uncertainty, and if you know one, like if you have exact information on say its position, then you can't know its momentum, for example, or vice versa. So if you had an uncertainty equals 0 for this. [...] Which if it is 0 it wouldn't be greater than $\hbar$ over 2, so you can never have the uncertainty being 0 because otherwise it wouldn't be greater than $\hbar$ over 2?" Even though the context of the interview is spin, the student reverts back to the more familiar uncertainty principle for position and momentum. Similarly to two of the test responses above, this student seems to assume that if one of the uncertainties is zero, then the product of two uncertainties could not be greater than zero, so that this would contradict the uncertainty principle and thus zero uncertainty is not possible. This idea is not correct, as e.g. a position eigenstate with zero position uncertainty has an infinite momentum uncertainty.

Another student in the interviews incorrectly thought that the uncertainty product of two spin components must always be greater than zero. For a definite $S_{x}$ with $\Delta S_{x}=0$, they could not reconcile this result with the fact that the outcomes of $\pm \hbar / 2$ imply that the second spin component could not have infinite uncertainty.

\section{DISCUSSION AND CONCLUSIONS}

The results in section III show that in the context of discrete probability distributions some students confuse quantum uncertainty with errors due to imperfections in the measurement apparatus (section III A). Only a minority of students sketched the uncertainty region from the expectation value outwards and thus related the uncertainty to the mean of the probability distribution (section III B). Some students thought that a quantum uncertainty of zero is not possible, or could not reconcile this result with uncertainty relations be- tween incompatible observables (section III C). Notably, very few students $(<5 \%)$ had problems calculating the quantum uncertainty in the activity and the test question. In contrast, there were substantial difficulties with the visual and conceptual interpretation of this result.

Müller and Wiesner's study with school students [3] and Ayene et al.'s study with undergraduate university students [5] found that some students incorrectly interpret Heisenberg's Uncertainty Principle between position and momentum in terms of measurement errors. We here see a similar difficulty (section III A), but now in the context of discrete probability distributions and depicted visually in terms of errors around each of the discrete outcomes.

Not relating uncertainty to the expectation value of the distribution (section III B) may be a difficulty that is specific to discrete or asymmetric probability distributions. For example, when asking students to sketch the uncertainty region for a continuous symmetric distribution such as a free particle Gaussian wave packet, our students seem to have no difficulty correctly sketching this uncertainty region from the peak (e.g. the expectation value of position for the position probability distribution) outwards.

The incorrect idea that quantum uncertainty can never be zero (section IIIC) was in some cases due to incorrect interpretations of uncertainty relations between incompatible observables. This difficulty may also be due to confusing quantum uncertainty with errors due to imperfections in the measurement apparatus. Investigating this difficulty further in terms of these two ways of thinking is an area for future work.

The results in this study have implications for instruction, in terms of a need to support students in developing correct visual and conceptual understanding of quantum uncertainty. We are currently developing a simulation-tutorial activity to support student understanding of this topic.

\section{ACKNOWLEDGMENTS}

The authors would like to thank all students that participated in these studies and provided feedback. We thank the University of St Andrews for funding the development of simulations.
[1] E. Marshman and C. Singh, Framework for understanding the patterns of student difficulties in quantum mechanics, Phys. Rev. ST Phys. Educ. Res. 11, 020119 (2015).

[2] E. Marshman and C. Singh, Investigating and improving student understanding of the expectation values of observables in quantum mechanics, Eur. J. Phys. 38, 045701 (2017).

[3] R. Müller and H. Wiesner, Teaching quantum mechanics on an introductory level, Am. J. Phys. 70, 200 (2002).

[4] C. Singh, Interactive learning tutorials on quantum mechanics,
Am. J. Phys. 76, 400 (2008).

[5] M. Ayene, J. Kriek, and B. Damtie, Wave-particle duality and uncertainty principle: Phenomenographic categories of description of tertiary physics students' depictions, Phys. Rev. ST Phys. Educ. Res. 7, 020113 (2011).

[6] K. Krijtenburg-Lewerissa, H. J. Pol, A. Brinkman, and W. R. van Joolingen, Insights into teaching quantum mechanics in secondary and lower undergraduate education, Phys. Rev. Phys. Educ. Res. 13, 010109 (2017). 
[7] M. M. Stein, C. White, G. Passante, and N. G. Holmes, Student interpretations of uncertainty in classical and quantum mechanics experiments, in 2019 Physics Education Research Conference Proceedings [Provo, UT, July 24-25, 2019], edited by Y. Cao, S. Wolf, and M. B. Bennett (2020) p. 573.

[8] E. M. Stump, C. L. White, G. Passante, and N. G. Holmes, Student reasoning about sources of experimental measurement uncertainty in quantum versus classical mechanics, in 2020 Physics Education Research Conference Proceedings Virtual Conference, July 22-23, 2020], edited by S. Wolf, M. B. Bennett, and B. W. Frank (2020) p. 527.

[9] G. Passante and A. Kohnle, Enhancing student visual under- standing of the time evolution of quantum systems, Phys. Rev. Phys. Educ. Res. 15, 010110 (2019).

[10] A. Kohnle, S. E. Ainsworth, and G. Passante, Sketching to support visual learning with interactive tutorials, Phys. Rev. Phys. Educ. Res. 16, 020139 (2020).

[11] QuVis Quantum Mechanics Visualization Project, Uncertainty of spin measurement outcomes, https: //www.st-andrews.ac.uk/physics/quvis/simulations_html5/ sims/uncertainty/measurement-outcome-uncertainty.html (Accessed May 27, 2021). 